\title{
Kontinuierliche Messung der katalytischen Aktivität von Adenosindesaminase mit der pH-Stat-Methode
}

\author{
Von H. Garth \\ Abteilung für Nuklearmedizin und Medizinische Physik, Fachbereich 4 Klinische Medizin der Universität \\ des Saarlandes, und \\ E. Zoch \\ Fachrichtung Physiologische Chemie, Fachbereich 3 Theoretische Medizin der Universität des Saarlandes, \\ Homburg/Saar
}

(Eingegangen am 6. April/25. Juli 1984)

Zusammenfassung: Bei der enzymatischen Desaminierung von Adenosin zu Inosin entsteht Ammoniak, welches einen $\mathrm{pH}$-Anstieg im Reaktionsgemisch bewirkt. Der $\mathrm{pH}$-Wert wird durch die $\mathrm{pH}$-Stat-Methode bei einem bestimmten Wert konstant gehalten, indem pH-Änderungen durch kontinuierliche Zugabe von Säure (bzw. Lauge) zum Versuchsansatz ausgeglichen werden. Die theoretischen Grundlagen der pH-Stat-Methode sowie ihre Grenzen zur Messung enzymatischer Desaminierungsreaktionen werden dargelegt. Für den $\mathrm{pH}$ Bereich von 6,4 bis 8,5 wird ein Korrekturfaktor abgeleitet und berechnet.

Die empfindliche pH-Stat-Methode ermöglicht die kontinuierliche und registrierende Bestimmung der Adenosindesaminase-Aktivität in stark gefärbten oder trüben biologischen Proben.

The continuous measurement of adenosine deaminase activity by the $\mathrm{pH}$-stat-method

Summary: The enzymatic deamination of adenosine to inosine produces ammonia, which causes a $\mathrm{pH}$-increase of the reaction mixture. The $\mathrm{pH}$-stat method is based on the continuous addition of protons to keep the $\mathrm{pH}$ at a constant value. The theoretical principles are discussed. The quantitative limits of the assay and the effect of changing the $\mathrm{pH}$ were investigated. A correction factor was derived and calculated for the $\mathrm{pH}$ range 6.4 to 8.5. This sensitive method allows the continuous recording of the adenosine deaminase activity in strongly coloured or turbid biological samples.

\section{Einfuihrung}

Das Enzym Adenosindesaminase (Adenosin-Aminohydrolase EC 3.5.4.4) (1), welches die hydrolytische Abspaltung der Aminogruppe von Adenosin bzw. von 2'-Desoxyadenosin unter Ammoniakbildung katalysiert, hat im Rahmen des Nukleinsäurestoffwechsels bzw. des Abbaus von Purinderivaten besondere Funktionen zu erfüllen (2) und scheint auch eine zentrale Rolle in der Vermittlung der Immunantwort zu spielen (3). Zur quantitativen Be- stimmung der katalytischen Aktivität von Adenosindesaminase stehen mehrere Methoden $(4,5,6,7)$ zur Verfügung, die sich hinsichtlich Präzision, Spezifität, apparativem Aufwand und Zeitbedarf unterscheiden. In der vorliegenden Arbeit werden neben der praktischen Durchführung vor allem die theoretischen Grundlagen der pH-Stat-Methode, ihre Möglichkeiten, und Grenzen zur kontinuierlichen Messung der enzymatischen Desaminierung von Adenosin bzw. 2'-Desoxyadenosin dargelegt. 


\section{Prinzip der Methode}

Bei der durch die Adenosindesaminase katalysierten hydrolytischen Desaminierung von Adenosin zu Inosin (Gl. 1) wird für jedes Mol umgesetztes Substrat $1 \mathrm{Mol}$ Ammoniak frei:

$$
\text { Adenosin }+\mathrm{H}_{2} \mathrm{O} \rightarrow \text { Inosin }+\mathrm{NH}_{3}(\mathrm{Gl} .1)
$$

Da es sich bei dem freigesetzten Ammoniak um eine schwache Base im Sinne Brönstedt's handelt, tritt eine Umsetzung mit Wasser ein (Gl.2):

$$
\mathrm{NH}_{3}+\mathrm{H}_{2} \mathrm{O} \rightarrow \mathrm{NH}_{4}^{+}+\mathrm{OH}^{-}
$$

Durch die Bildung der Hydroxylionen steigt der $\mathrm{pH}-$ Wert an, wenn man ihn nicht mit geeigneten Mitteln konstant hält, etwa durch Pufferung des Reaktionsansatzes oder; durch Zugabe einer entsprechenden Menge von Protonen. Im zweiten Fall, welcher dem pH-Stat-Verfahren entspricht, ist die erforderliche Protonenmenge gleich der umgesetzten AdenosinMenge, vermindert um den Anteil des Ammoniaks, der bei dem gegebenen $\mathrm{pH}$-Wert als freie Base vorliegt. Dieser Anteil läßt sich nach der Gleichung von Henderson und Hasselbalch (Gl.3) berechnen:

$$
\mathrm{pH}=\mathrm{pK}_{\mathrm{a}_{\text {Ammonium }}}+\log \frac{\left[\mathrm{NH}_{3}\right]}{\left[\mathrm{NH}_{4}^{+}\right]}
$$

Der $\mathrm{pK}_{\mathrm{a}}$-Wert von Ammonium beträgt 9,25.

Setzt man

$$
\left[\mathrm{NH}_{3}\right]+\left[\mathrm{NH}_{4}^{+}\right]=1
$$

so ergibt sich für die Menge $\mathrm{A}$ an Protonen, die insgesamt bei der Umsetzung von $1 \mathrm{Mol}$ Adenosin benötigt werden,

$$
A=1 /\left(1+10^{\mathrm{pH}-9,25}\right)
$$

Die Auftragung dieser Funktion liefert die Kurve a der Abbildung 1.

Nun müssen aber nicht alle diese Protonen dem System von außen zugeführt werden, denn das bei der Reaktion (Gl. 1) entstandene Inosin ist mit einem $\mathrm{pK}_{\mathrm{a}}$-Wert von 8,8 (8) als schwache Säure zu sehen und stellt je nach $\mathrm{pH}$-Wert einen mehr oder minder großen Teil der benötigten Protonen zur Verfügung.

$$
\text { Inosin } \rightarrow \text { Inosinat }^{-}+\mathrm{H}^{+}
$$

Dieser vom Reaktionsprodukt Inosin beigesteuerte Anteil an Protonen ist ebenfalls nach HendersonHasselbalch $\mathrm{zu}$ berechnen:

$$
\mathrm{pH}=\mathrm{pK}_{\mathrm{a}_{\mathrm{inosin}}}+\log \frac{\left[\text { Inosinat }^{-}\right]}{\left[\text {Ind́din }^{-}\right]}
$$

Setzt man wiederum

$$
[\text { Inosin }]+\left[\text { Inosinat }^{-}\right]=1
$$

so ergibt sich die Menge $B$ der bei der Hydrolyse von $1 \mathrm{Mol}$ Adenosin zu Inosin zur Verfügung gestellten Protonen:

$$
\mathrm{B}=1 /\left(1+10^{8,8-\mathrm{pH}}\right)
$$

Die Auftragung dieser Funktion liefert die Kurve $b$ der Abbildung 1. Die Differenz A - B muß dann als starke Säure von außen zugegeben werden, wenn während der Reaktion der $\mathrm{pH}=$ Wert konstant bleiben soll.

Entsprechend kann ein pH-abhängiger Faktor berechnet werden, mit dem der gemessene Säureverbrauch zu korrigieren ist, wenn man die tatsächlich umgesetzte Adenosin-Menge aus dem gemessenen Säureverbrauch ermitteln will:

$$
\mathrm{F}=1 /(\mathrm{A}-\mathrm{B})
$$

Für den physiologischen pH-Bereich wurde der Korrekturfaktor F entsprechend $(\mathrm{Gl} .8,9)$ und $(\mathrm{Gl} .10)$ berechnet und tabelliert (Tab. 1).

Tab. 1. Korrekturfaktor $\mathrm{F}$ für den $\mathrm{pH}-\mathrm{Bereich}$ von 6,4 bis 8,5 . pH 6,8 entspricht dem pH-Optimum der Adenosindesaminase aus Kalbsthymus (9).

\begin{tabular}{llll}
\hline pH & F & pH & F \\
\hline 6,4 & 1,005 & 7,5 & 1,070 \\
6,5 & 1,007 & 7,6 & 1,088 \\
6,6 & 1,009 & 7,7 & 1,112 \\
6,7 & 1,011 & 7,8 & 1,143 \\
6,8 & 1,014 & 7,9 & 1,183 \\
6,9 & 1,017 & 8,0 & 1,235 \\
7,0 & 1,022 & 8,1 & 1,303 \\
7,1 & 1,027 & 8,2 & 1,394 \\
7,2 & 1,034 & 8,3 & 1,518 \\
7,3 & 1,044 & 8,4 & 1,691 \\
7,4 & 1,055 & $.8,5$ & 1,941 \\
\hline
\end{tabular}


Dabei ist zu sehen, daß bei den für Messungen am häufigsten benutzten $\mathrm{pH}$-Werten von 6,8 und 7,4 die Korrektur nur 1,4\% bzw. 5,5\% des Meßwertes ausmacht, bei pH 8,5 aber schon über $60 \%$. Schließlich wird etwa bei $\mathrm{pH}>9$ die Messung von der Theorie her unmöglich, da alle vom Ammoniak verbrauchten Protonen vom Inosin geliefert werden $(A=B)$.

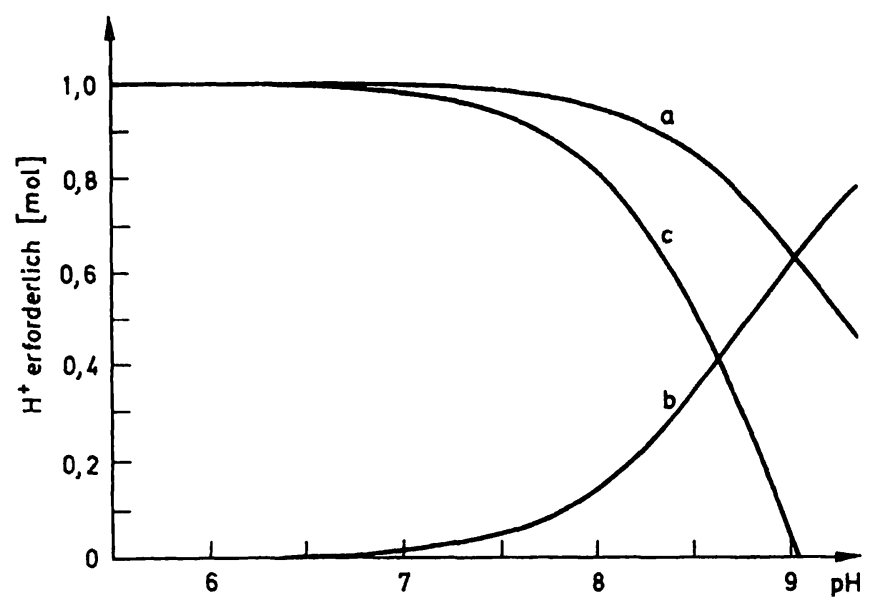

Fig. 1. Bei der enzymatischen Desaminierung von 1 Mol Adenosin zur Konstanthaltung des $\mathrm{pH}$-Wertes benötigte Protonenmenge in Abhängigkeit vom pH-Wert (Kurve c); Kurven $a$ und $b$ siehe Text.

Prinzipiell gelten diese Betrachtungen auch für Adenosinderivate und andere desaminierbare Substrate, wenn die entsprechenden $\mathrm{pK}_{\mathrm{a}}$-Werte im gleichen Bereich liegen. Bei Abweichung der zugrunde gelegten pK=Werte (auch bei Änderungen der Meßtemperatur) muß gegebenenfalls eine erneute Berechnung des Korrekturfaktors $F$ entsprechend $(\mathrm{Gl} .5,9)$ und (Gl. 10) erfolgen.

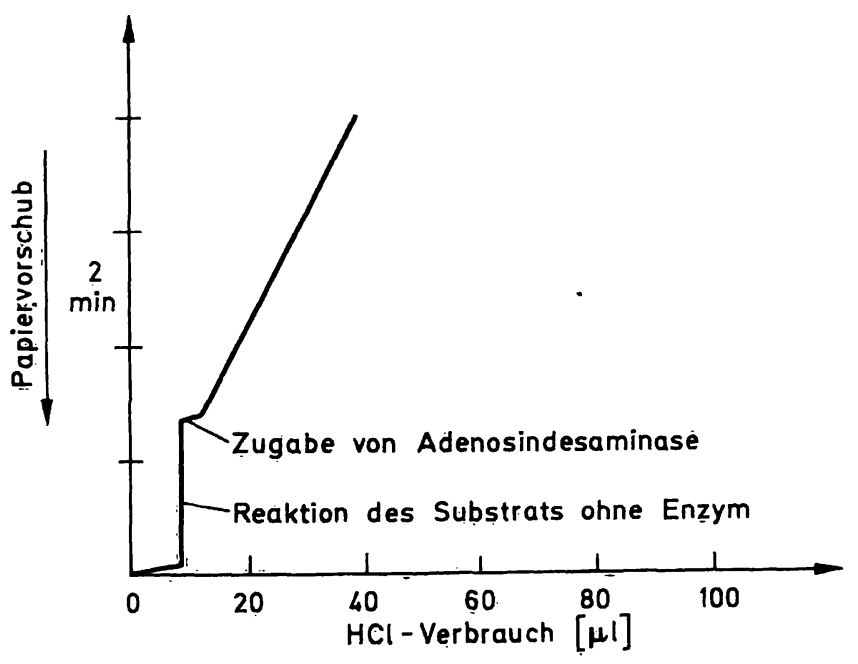

Fig. 2. Kontinuierliche Messung der katalytischen Aktivität von Adenosindesaminase nach dem pH-Stat-Verfahren (schematisch).
Vergleichende Messungen haben gezeigt, daß unterhalb eines $\mathrm{pH}-$ Wertes von 9 der durch Entweichen von Ammoniak in die Luft entstandene Fehler vernachlässigt werden kann. Einer Beeinflussung des Meßergebnisses durch Aufnahme von $\mathrm{CO}_{2}$ aus der Luft kann durch Spülung des über der Probe befindlichen Gasraumes mit einem inerten Gas (Stickstoff, Argon) begegnet werden.

\section{Praktische Durchfiihrung}

Die Messung der katalytischen Aktivität von Adenosindesaminase erfolgt in einer Apparatur der Fa. Metrohm, Herisau.

Im einzelnen tragen die Geräte folgende Typenbezeichnungen:

- Präzisions-pH-Meter E 510

- Regler: Impulsomat E 473

- Motorbürette E 415 mit Ansatz V = 0,2 ml

- Schreiber E 419

- Reaktionsgefäß, V $=1,0 \mathrm{ml}$, thermostatisierbar

- Magnetrührer E $332 \mathrm{H}$

- Glaselektrode: Einstab-Meßkette EA 47

Zur Messung wird das Reaktionsgefä $\beta$ auf die gewünschte Meßtemperatur thermostatisiert. Die Bürette enthält $\mathrm{HCl}(0,01$ $\mathrm{mol} / \mathrm{l})$. Nach Vorlage von $1,0 \mathrm{ml}$ der vorthermostatisierten Substrat-Lösung $(0,01 \mathrm{~mol} / \mathrm{l})$ wird der pH-Wert (z.B. pH 6,8) zunächst mit $\mathrm{HCl}(0,1 \mathrm{~mol} / \mathrm{l})$ bzw. $\mathrm{NaOH}(0,1 \mathrm{~mol} / \mathrm{l})$ mit Hilfe einer Mikroliterspritze auf einen Wert eingestellt, der etwa $0,01 \mathrm{pH}$ oberhalb des Meß-pH-Wertes liegt. Unmittelbar nach Zugabe eines geeigneten Volumens der zu messenden Probe (z.B. $1 \mu$ l oder $10 \mu \mathrm{l})$ wird die Messung gestartet. Der Zusatz der im Reaktionsverlauf benötigten Säure erfolgt dann automatisch. Auf eine optimale Einstellung der Gerätekombination (Empfindlichkeit des Reglers, Abhängigkeit der zugesetzten Säure-Menge von der Soll-Ist-Abweichung, Geschwindigkeit der Registrierung) muB geachtet werden.

Aus dem pro Zeiteinheit verbrauchten Volumen an $\mathrm{HCl}(0,01$ $\mathrm{mol} / \mathrm{I}$ ) (Tangente an die registrierte Kurve zu Reaktionsbeginn) kann die Aktivität der Adenosindesaminase unter Berücksichtigung des Korrekturfaktors berechnet werden (Abb.2).

\section{Diskussion}

Der Vorteil des beschriebenen pH-Stat-Verfahrens gegenüber der oft verwendeten kontinuierlichen spektrophotometrischen Methode (4) liegt darin, $\mathrm{da} \hat{B}$ noch geringe katalytische Konzentrationen der Adenosindesaminase (Adenosinumsatz bis zu $10^{-5}$ $\mathrm{mol} / \mathrm{A} \cdot \mathrm{min}$ ) kontinuierlich und registrierend auch in stark gefärbten bzw. trüben Lösungen (z. B. Erythrocytenhämolysat) oder in optisch dichten Extrakten (z.B. Organextrakte, Zellfraktionen) $(10,11)$ ohne zusätzlichen Geräteaufwand gemessen werden können. Die pH-Stat-Methode erlaubt auch die 
Messung der Adenosindesaminase bei Substratsättigung $\left(100 \times K_{\mathrm{m}_{\text {Adensin }}}\right)$, was mit dem optischen Bestimmungsverfahren nicht möglich ist. Bei der spektrophotometrischen Messung der katalytischen Aktivität von Adenosindesaminase, welche auf dem unterschiedlichen spektralen Verhalten von Adenosin und Inosin beruht, kann nämlich nur noch eine Adenosinkonzentration von $130 \mu \mathrm{mol} / 1\left(<K_{\mathrm{m}}\right.$ für Adenosin) verwendet werden, wenn die Gesamtabsorbanz des Meßansatzes unter 2 bleiben soll. Dies wird auch durch die Befunde von Murphy et al. bestätigt (12). i

\section{Literatur}

1. Enzyme Nomenclature, Recommendations (1978) of the Nomenclature Committee of the International Union of Biochemistry (IUB), Academic Press, Inc. New York 1979.

2. Henderson, J. F., Lowe, J. K. \& Barankiewicz, J. (1977) In: Purine and Pyrimidine Metabolism, Ciba Foundation Symposium 48, 3-21.

3. Thompson, L. F. \& Seegmiller, J. E. (1979) Adv. Enzymol. $51,167-210$.

4. Kalckar, H. M. (1947) J. Biol. Chem. 167, 429-433.

5. Tritsch, G. L. \& Mittelman, A. (1975) Biochem. Med. 12, $66-71$.

6. Rogler-Brown, R. P., Agarwal, R. E. \& Parks, R. E. (1978) Biochem. Pharmacol. 27, 2289.

7. Daddona, P. E., Frohman, M. A. \& Kelley, W. N. (1979) J. Clin. Invest. 64, 798-803

8. Handbook of Biochemistry, Chemical Rubber Company, 2nd. ed. 1970

9. Garth, H. (1982) Dissertation Math. Naturwiss. Fak. (1982) Universität des Saarlandes.

10. Zoch, E. (1980) Erfahrungsheilkunde 29, 105-108.

11. Zoch, E. \& Häge, R. (1977) Zbl. Gynäkol. 99, 337-342.

12. Murphy, J., Baker, D. C., Behling, C. \& Turner, R. A. (1982) Analyt. Biochem. 122, 328-337.

Prof. Dr. E. Zoch

Fachrichtung Physiologische Chemie Fachbereich 3 Theoretische Medizin der Universität dẹ Saarlandes D-6650 Homburg/Saär 